Article

\title{
Evidence of Intersubband Linewidth Narrowing Using Growth Interruption Technique
}

\author{
Ngoc Linh Tran 1,*(D), Giorgio Biasiol ${ }^{2}$, Arnaud Jollivet ${ }^{1}$, Alberto Bertocci ${ }^{1}$, François H. Julien ${ }^{1}$, \\ Jean-Michel Manceau ${ }^{1, *}$ and Raffaele Colombelli ${ }^{1}$ \\ 1 Centre for Nanosciences and Nanotechnologies, CNRS UMR 9001, Université Paris-Sud, Université \\ Paris-Saclay, 91120 Palaiseau, France; arnaud.jollivet@u-psud.fr (A.J.); albertobertocci@live.it (A.B.); \\ francois.julien@u-psud.fr (F.H.J.); raffaele.colombelli@u-psud.fr (R.C.) \\ 2 Laboratorio TASC, CNR-IOM, Area Science Park, I34149 Trieste, Italy; biasiol@iom.cnr.it \\ * Correspondence: ngoc-linh.tran@u-psud.fr (N.L.T.); jean-michel.manceau@u-psud.fr (J.-M.M.)
}

Received: 8 February 2019; Accepted: 27 March 2019; Published: 1 April 2019

Abstract: We report on the systematic study of two main scattering mechanisms on intersubband transitions, namely ionized impurity scattering and interface roughness scattering. The former mechanism has been investigated as a function of the dopants position within a multiple GaAs/AlGaAs quantum well structure and compared to the transition of an undoped sample. The study on the latter scattering mechanism has been conducted using the growth interruption technique. We report an improvement of the intersubband (ISB) transition linewidth up to $11 \%$ by interrupting growth at GaAs-on-AlGaAs interfaces. As a result, the lifetime of intersubband polaritons could be improved up to $9 \%$. This leads to a reduction of $17 \%$ of the theoretical threshold intensity for polaritonic coherent emission. This work brings a useful contribution towards the realization of polariton-based devices.

Keywords: Quantum wells; Intersubband transitions; Growth interruption; Modulation doping; Polaritons

\section{Introduction}

Intersubband transitions (ISBT) in square semiconductor quantum wells (QW) are the building blocks of mid-infrared/terahertz optoelectronic devices, such as quantum cascade lasers (QCL) [1], quantum well infrared photodetectors (QWIP) [2], second harmonic generation [3], and potential polaritonic coherent emitters [4,5]. The ISBT lifetime is governed by the different relaxation mechanisms of electrons and is relevant for both applied and fundamental physics. Among the various relaxation mechanisms of electrons in confined electronic states, experiments suggest that elastic processes, such as interface roughness and ionized impurities, are the major axes of improvement to reach the intrinsic linewidth limitation due to phonons [6-8]. Prior to the tremendous success of the QCL, the improvement of ISBT lifetime was an active field of research. Although its interest has decreased, it remains of importance especially in the perspective of intersubband polaritonic lasers [5]. Such polaritonic coherent emitter is based on final state stimulation, and it was proposed to exploit longitudinal optical (LO) phonon-polariton interaction as the main spontaneous scattering mechanism. The first study of phonon-polariton scattering was reported several years ago with electrically injected devices [9], and more recently phonon-polariton scattering mediated polaritonic emission was demonstrated under an optical pumping scheme [10]. The threshold intensity for final state stimulation can be estimated using the following expression:

$$
I_{t h}=\frac{\hbar^{2} \Gamma_{L O} \Gamma_{U P} \Gamma_{L P} \omega_{U P} \varepsilon_{\rho} N_{Q W}}{4 \pi\left|\alpha^{i}\right|^{2}\left|\alpha^{f}\right|^{2} \omega_{L O} f e^{2} L_{Q W}}
$$


where $I_{t h}$ is the threshold pump intensity, $\Gamma_{L O}$ is the damping rate of the LO-phonon, $\Gamma_{U P / L P}$ are linewidths of upper and lower polaritons, $\omega_{U P}$ is the pump photon frequency, $\varepsilon_{\rho}$ is the relative dielectric constant due to phonon. $N_{Q W}$ is the number of quantum wells, $\alpha^{i, f}$ are the matter-part Hopfield coefficients of the initial and final polaritonic states, $\omega_{L O}$ is the LO phonon frequency, $f$ is a numerical factor summarizing the intrasubband nature of the scattering process, and $L_{Q W}$ is the QW widths.

A key aspect towards the demonstration of an intersubband (ISB) polaritonic laser is to reduce the lasing threshold intensity which is around $1 \mathrm{MW} / \mathrm{cm}^{2}$ for a $36 \mathrm{QWs}$ structure [10]. From the above expression, it appears that both polariton lifetime (upper and lower state) are a lever towards this goal. The polariton lifetime goes as the inverse of the polaritonic linewidth $\left(1 / \Gamma_{U P, L P}\right)$, itself governed by the linewidth of ISB plasmon and by the linewidth of the resonant optical mode, respectively, and weighted by the Hopfield coefficients [11]:

$$
\Gamma_{L P / L P}=\left|\alpha_{U P / L P}\right|^{2} \Gamma_{I S B}+\left|\beta_{U P / L P}\right|^{2} \Gamma_{c a v}
$$

The cavity linewidth $\left(\Gamma_{\text {cav }}\right)$ is mainly affected by the Ohmic losses stemming from the presence of the metallic bottom layer and grating top layer. Optimization of the geometrical aspects of the cavity or the use of different confining material can be used to improve the cavity lifetime but are out of the scope of this paper $[12,13]$. As already mentioned, the broadening of ISBT $\left(\Gamma_{I S B}\right)$ is mainly governed by ionized impurities, interface roughness scattering, and phonon scattering. Another cause of broadening comes from the presence of multiple quantum wells (MQW) that can be a source of inhomogeneities (well-to-well thickness variation, or non-uniform distribution of doping across the quantum wells). Nevertheless, it has been already demonstrated theoretically [14] and experimentally [15] that the strong coupling regime is insensitive to inhomogeneity to the extent that the coupling frequency $\left(\Omega_{\text {Rabi }}\right)$ is larger than the inhomogeneous broadening $\left(\gamma_{\text {inho }}\right)$.

In the present work, we first study with transmission spectroscopy the ionized impurities scattering mechanism by tuning the positions of the Si-donor layers. The measured ISBT linewidths are compared to the one obtained from an undoped sample that is de facto free from ionized impurities scattering. Secondly, we study the interface roughness scattering by examining the effect of the growth interruption on the linewidth of the ISBT for both interfaces. Finally, we theoretically investigate the impact of the interruption growth technique on the polaritonic lifetime and estimate how much it could reduce the lasing threshold intensity of an ISB polaritonic laser.

\section{Samples Description and Experimental Setup}

\subsection{Samples Description}

The MQW structures used in this study were initially designed for the demonstration of intersubband polaritons in metal-insulator-metal (MIM) cavities $[10,16]$. They have been grown by molecular beam epitaxy on Semi-insulating GaAs. Each period consists of an 8.3-nm-thick GaAs well and a 20-nm-thick $\mathrm{Al}_{0.33} \mathrm{Ga}_{0.67} \mathrm{As}$ barrier. Schrödinger-Poisson simulations (without taking into account the Coulomb interactions) predict the fundamental ISB transition at an energy of $116 \mathrm{meV}$.

The first set of samples aims to study the ISBT broadening mechanism of ionized impurities. Two doped samples and one nominally undoped sample were grown. The doped samples consist of 35 repetitions of QWs. In the first (HM3770), the wells were volume-doped with nominal concentration $\mathrm{n}_{2 \mathrm{D}}=1.05 \times 10^{11} \mathrm{~cm}^{-2}$. The second (HM3818) was remotely $\delta$-doped at the center of the barriers with $\mathrm{n}_{2 \mathrm{D}}=1.02 \times 10^{11} \mathrm{~cm}^{-2}$ (modulation doping). The nominally undoped sample (HM4105) consists of 87 repetitions. Here, the number of quantum wells is higher in order to increase the absorption of the ISBT since the number of excited charges is relatively low in comparison to a doped one. The details of the samples are presented in Table 1. 
Table 1. The first set of samples for studying the broadening mechanism of ionized impurities.

\begin{tabular}{cccccc}
\hline Sample & Detail & $\begin{array}{c}\text { Doping } \\
\left(\mathbf{c m}^{-2}\right)\end{array}$ & $\begin{array}{c}\text { Number of } \\
\text { QWs }\end{array}$ & $\begin{array}{c}\text { Intersubband } \\
\text { Transition (meV) }\end{array}$ & $\begin{array}{c}\text { Linewidth } \\
(\mathbf{m e V})\end{array}$ \\
\hline HM3770 & Volume doping & $1.05 \times 10^{11}$ & 35 & 129.4 & 13.1 \\
HM3818 & Modulation doping & $1.02 \times 10^{11}$ & 35 & 119.7 & 6.3 \\
HM4105 & Nominally undoped & & 87 & 127 & 6.4 \\
\hline
\end{tabular}

The second set of samples, including eight samples (grown on the same batch), targets to study the ISBT broadening mechanism of interface roughness. All the samples were grown in the same configuration with 38-repetitions, and nominally doped to $\mathrm{n}_{2 \mathrm{D}}=1.02 \times 10^{12} \mathrm{~cm}^{-2}$ at the center of the barriers. The growth interruption was applied during the process for either or both interfaces (GaAs-on-AlGaAs and AlGaAs-on-GaAs) for 30, 60, and $120 \mathrm{~s}$ of interruption time. The details of the second sample set are listed in Table 2.

Table 2. The second set of samples for studying the broadening mechanism of interface roughness.

\begin{tabular}{cccc}
\hline Sample & Detail & Doping $\mathbf{( c m}^{-\mathbf{2}} \mathbf{)}$ & Number of QWs \\
\hline HM4046 & 0 second (s.) growth interruption (g.i) & $1.02 \times 10^{12}$ & 38 \\
HM4048 & 30 s. g.i on AlGaAs/GaAs & $1.02 \times 10^{12}$ & 38 \\
HM4049 & 60 s. g.i on AlGaAs/GaAs & $1.02 \times 10^{12}$ & 38 \\
HM4042 & 120 s. g.i on AlGaAs/GaAs & $1.02 \times 10^{12}$ & 38 \\
HM4043 & 30 s. g.i on GaAs/AlGaAs & $1.02 \times 10^{12}$ & 38 \\
HM4044 & 60 s. g.i on GaAs/AlGaAs & $1.02 \times 10^{12}$ & 38 \\
HM4045 & 120 s. g.i on GaAs/AlGaAs & $1.02 \times 10^{12}$ & 38 \\
HM4014 & 120 s. g.i on both interfaces & $1.02 \times 10^{12}$ & 38 \\
\hline
\end{tabular}

\subsection{Experimental Setup}

In the case of doped samples, due to the high concentration of charge carriers in the QWs, the 1st QW subband is populated with electrons. We used a standard transmission spectroscopy approach to measure the ISB transition absorption. A 5-nm/100-nm-thick layer of Ti/ $\mathrm{Au}$ was first deposited on the sample's surface to maximize the field overlap with the active region. The samples were then shaped in a multi-pass waveguide structure by mechanical polishing to obtain $45^{\circ}$ double facets as depicted in Figure 1a [17]. The samples were mounted on copper blocks for measurements at $78 \mathrm{~K}$. The Globar source from a Fourier transform interferometer (Vertex 70, Bruker, Germany) was polarized and focused on the $45^{\circ}$-facet prism, and the transmitted signal was collected onto a Deuterated Triglycine Sulfate (DTGS) detector.

In the case of nominally undoped samples, the 1st QW subband is empty, and it is necessary to promote carriers from the valence band. A photo-induced absorption experiment has been implemented to this scope [18]. This sample was also shaped in a multipass waveguide configuration with double polished facets but without the top metallic layer in order to leave access to the active region for photo-excitation. A continuous wave pump laser ( $532 \mathrm{~nm}$ ) was used to photo-excite electrons from the valence band to the 1 st confined level of the conduction band. The probe beam (Globar source) was polarized and focused on the $45^{\circ}$ facet out of the FTIR and the collected signal sent to a mercury cadmium telluride (MCT) detector. The spectrum was recorded using a step scan technique and a synchronous detection scheme to improve the signal-to-noise ratio. 

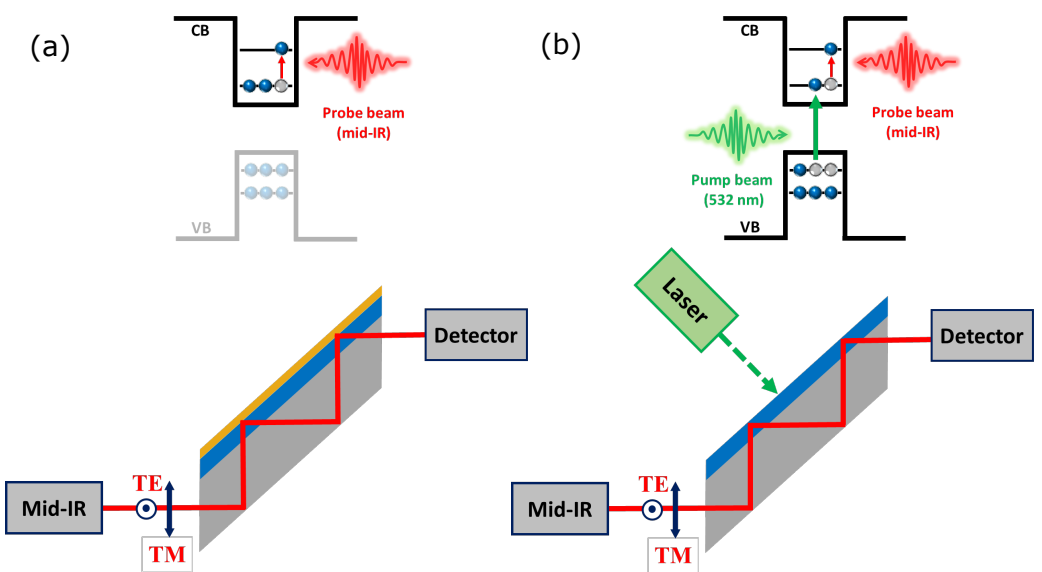

Figure 1. (a) (upper panel) Schematic of the absorption spectroscopy of Mid-Infrared (MIR) photons within a doped Quantum Well (QW). The 1st subband (conduction band) is filled with electrons and MIR photons are absorbed by the quantized states. (lower panel) Schematic of the experimental approach with a $45^{\circ}$ facets multipass prism. The sample is probed in both Transverse Electric (TE) polarization and Transverse Magnetic polarization (TM) (b) (Upper Panel) Schematic representation of the photo-induced absorption spectroscopy with nominally undoped QW. Electrons are promoted from the valence band up to the conduction band using a continuous wave laser at $532 \mathrm{~nm}$. MIR photons are then absorbed by the quantized states. (lower panel) Schematic of the experimental approach with a $45^{\circ}$ facets multipass prism illuminated by a CW laser.

\section{Results}

\subsection{Effect of the Ionized Impurities Scattering}

The transmission spectra of the doped samples (HM3770 and HM3818) recorded at $78 \mathrm{~K}$ are shown in Figure 2a. The black dots show the experimental data, while the red lines represent the Voigt fit. The Voigt fitting function is the most appropriate one when dealing with measurements that can exhibit both homogenous (Lorentzian shape) and inhomogeneous broadening (Gaussian shape) [15]. This function is a convolution in between Lorentz and Gaussian functions having the same peak frequency. The non-linear least squares fitting procedure then gauge the amount of Lorentzian weight versus the Gaussian one in order to obtain the most appropriate fit. The ISB transition energy of the modulation doped sample is red-shifted by $9.7 \mathrm{meV}$ with respect to the one of the samples doped within the well. This is the result of the Hartree potential due to donors and charges separation. The self-consistent potential applied to the conduction band effectively reduces the depth of the QW and, in turn, compensates for the depolarization shift induced by the plasma frequency [17,19-21].
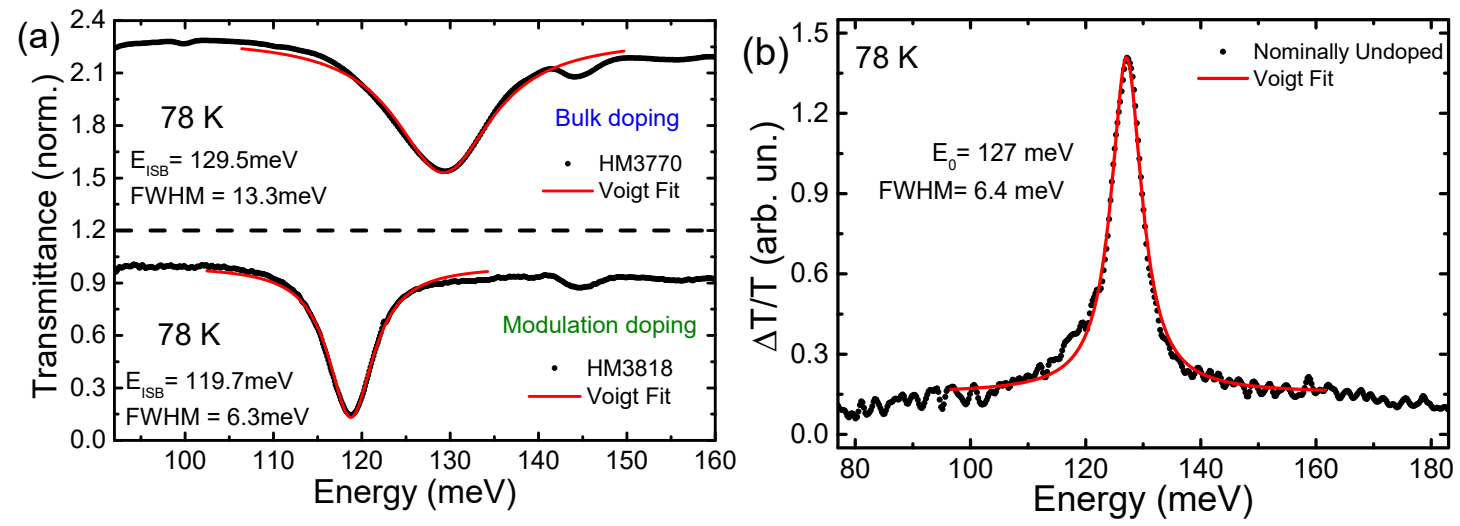

Figure 2. (a) Transmittance spectra of the sample doped within the well (upper panel) and the modulation doped sample at $78 \mathrm{~K}$ (lower panel). The red curves are the Voigt fitting functions. (b) The photo-induced absorption spectrum of the nominally undoped sample at $78 \mathrm{~K}$ with its Voigt fit (red). 
To our interest, a line narrowing of factor two is observed between the modulation doped sample with a full width at half maximum (FWHM) of $6.3 \mathrm{meV}$ and the volume doped sample with a $13.1 \mathrm{meV}$ FWHM. The difference is due to the scattering of charges by ionized impurities within the QW region in the case of the volume doped sample, which is in good agreement with the literature [7].

To gain more knowledge on the influence of ionized impurities scattering, we measured the ISB transition of an undoped sample at $78 \mathrm{~K}$ using a photo-induced absorption spectroscopy technique. Such a sample is free from the ionized impurities scattering channel. Figure $2 \mathrm{~b}$ shows the absorption spectrum with an ISB transition at $127 \mathrm{meV}$. In such a scenario, electrons are promoted from the valence band up to the conduction band and then relax to the 1st confined level inside the QW. The charge density is very weak; thus we cannot consider the influence of the depolarization shift and attribute the different central frequency of the transition to drift in time of the Molecular Beam Epitaxy (MBE) system. X-ray diffractometry measurement attested a difference of $0.4 \mathrm{~nm}$ in the well's thickness in between these two samples, confirming the blue-shift of $8 \mathrm{meV}$ for HM4105. More interestingly, the ISBT FWHM is equal to $6.4 \mathrm{meV}$, which is comparable to the linewidth of the modulation-doped sample. This confirms that for relatively low-doped structures, if the donors are placed remotely in the barriers, impurity scattering weakly contributes to the broadening of the transition, similarly to low-temperature transport phenomena [22].

\subsection{The Improvement of the Interface Roughness by Growth Interruption}

The growth interruption technique has been successfully applied in the past at heterostructure interfaces to smooth them out [23]. Roughness at the interfaces of GaAs / AlGaAs multiple quantum wells was estimated to be of the order of one atomic layer $(\mathrm{a}=2.83 \AA$ ), with lateral periodicities of the order of $300 \AA[24,25]$. More importantly, it was reported to dominate the broadening of ISBT, especially for narrower wells $<10 \mathrm{~nm}[6,26]$. It is, therefore, crucial for our application to gauge the impact of interface roughness and to investigate possible countermeasures.

The influence of the growth interruption depends on many factors, including the types of interfaces (GaAs-on-AlGaAs or AlGaAs-on-GaAs), the Al-concentration of the barrier, the specific design of the multiple QW systems [27,28], the growth conditions, and the concentration of residual impurities in the MBE system. In the following, we study its influence when applied to either and both types of interfaces, AlGaAs-on-GaAs (named normal interface) and GaAs-on-AlGaAs (named inverted interface).

Here, we consider the second batch of samples, where we applied the growth interruption (g.i.) technique. All the transmission spectra were measured at $78 \mathrm{~K}$. The result of the growth interruption on the inverted interfaces, as a function of the interruption time, is represented in Figure 3a. We note a similar peak transition between the 0 second (s.)-Growth Interruption (g.i.) sample and the modulation doped sample of the first set. The sheet doping concentration is one order of magnitude higher for this second batch, demonstrating once again the balance between depolarization shift and the Hartree potential. More interestingly, the linewidths are reduced by $1 \mathrm{meV}$ for 30 s.-g.i. and 120 s.-g.i. samples. This phenomenon is similar to previous observations done by photoluminescence measurements $[23,27,29]$. As the inverted interface (GaAs-on-AlGaAs) is known to be rougher than the normal interface [30], the growth interruption favours the processes of diffusion and migration of atoms at the inverted interface, resulting in smoother interfaces and, in turn, narrower linewidths. The result of the growth interruption on the normal interfaces, as a function of the interruption time, is presented in Figure 3b. Here, the ISBT linewidths increase gradually with interruption time. The unfavorable influence of the growth interruption on the normal interfaces is probably due to potential accumulation of impurities on GaAs surfaces. The finding that the influence of the g.i. is different for top and bottom interfaces is in good agreement with previous observations $[27,28]$. 

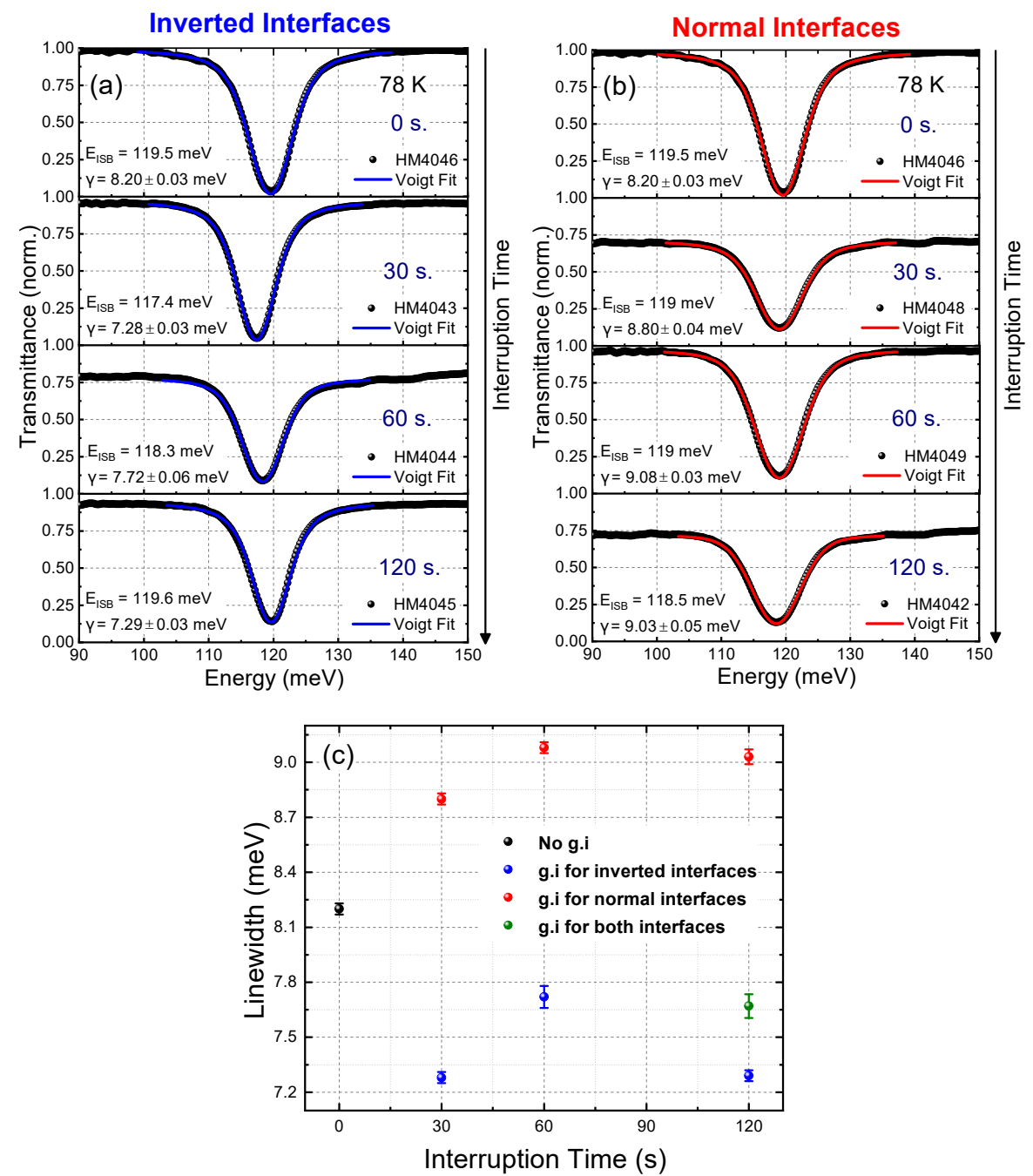

Figure 3. (a) ISB transmission spectra of the samples where the growth interruption was applied on the inverted interfaces $(0,30,60$, and $120 \mathrm{~s}$.). (b) ISB transmission spectra of the samples, where the growth interruption was applied on the normal interfaces $(0,30,60$, and $120 \mathrm{~s}$.). (c) The dependence of the ISBT linewidth on the growth interruption time.

To summarize the effect of g.i on both interfaces, we display the dependence of linewidth on g.i. times in Figure 3c. We observe clearly the gradual increase of ISB linewidth up to $11 \%$ when the growth interruption is applied for normal interfaces, while it decreases by $11 \%$ when applying the technique to the inverted ones. Additionally, in contrast to previous Photo-Luminescence measurements [27,28], the 120 s. -120 s. g.i. for both interfaces shows an ISBT broadening of $7.7 \mathrm{meV}$, which is close to the no g.i. value and suggests that the beneficial and detrimental effects of g.i. at the two interfaces tend to balance out. Finally, we anticipate that similar results could be obtained for another III-V material system, such as InGaAs/AlInAs, as similar interfaces smoothing led to a narrowing of excitons photoluminescence [31].

\section{The Influence of the Growth Interruption on the Strong Coupling Regime}

As we demonstrated the effect of the growth interruption on the ISB linewidth narrowing, we investigated the nature of the broadening induced by interface roughness. From a theoretical point of view, the nature of interface roughness scattering was first considered as purely homogeneous [24-26], and more recently as inhomogeneous [32]. Experimentally, it has been shown that the strong coupling regime is insensitive to inhomogeneity to a certain extent $\left(\gamma_{\text {inho }}<\Omega_{\text {Rabi }}\right)[15,33]$. Therefore, measuring 
the linewidth of the polaritons permits to probe the existence of inhomogeneity within the broadening of the ISB transition.

To evaluate the level of inhomogeneity in the studied samples, we follow the same experimental approach by processing one of them in MIM cavity architecture [16]. We embedded the sample HM4045 (thickness $1.19 \mu \mathrm{m}$ ) in a double metal cavity consisting of a back-plane gold mirror and a top patterned gold grating with a period of $4.35 \mu \mathrm{m}$ and a filling factor of $85 \%$. Figure $4 \mathrm{~b}$ shows the schematics of the cavity-ISB polaritonic device. The ISBT linewidth was also characterized at $300 \mathrm{~K}$ by transmission spectroscopy. Using the Voigt function fitting, we got the ISBT peak at $117.1 \mathrm{meV}$ with an FWHM of $9.7 \mathrm{meV}\left(\Gamma_{\text {ISB }}\right.$ ) (Figure $4 \mathrm{a}$ ). We then probed the polaritonic dispersion with an angle-resolved unit between $13^{\circ}$ and $73^{\circ}$ with an angular resolution of $2^{\circ}$. The reconstructed dispersion of the cavity-ISB polaritons presents clearly the two polaritonic branches, lower polariton (LP) and upper polariton (UP) (Figure 4c). Such design of the ISB polaritonic device offers an anti-crossing at $31^{\circ}$, where the light-matter fraction is $50 \%-50 \%$. Therefore, the linewidths of lower and upper polaritons are gauged from the reflectivity spectrum at this specific angle and are equal to $6.1 \mathrm{meV}\left(\Gamma_{\mathrm{LP}}\right)$ and $6.3 \mathrm{meV}\left(\Gamma_{\mathrm{LP}}\right)$, respectively. Furthermore, the cavity linewidth can be estimated on the asymptotic part of the dispersion where the matter fraction is extremely small. At an angle of $73^{\circ}$, we estimate the linewidth of the cavity to be $3.3 \mathrm{meV}\left(\Gamma_{\mathrm{Cav}}\right)$. Consequently, the polariton linewidth, $\Gamma_{\mathrm{LP}}$ and $\Gamma_{\mathrm{UP}}$, is in the order of the average calculated value, $\left(\Gamma_{\mathrm{Cav}}+\Gamma_{\mathrm{ISB}}\right) / 2=6.5 \mathrm{meV}$, expected at zero-detuning. This result reveals that the level of inhomogeneities is quasi-inexistent in our samples.
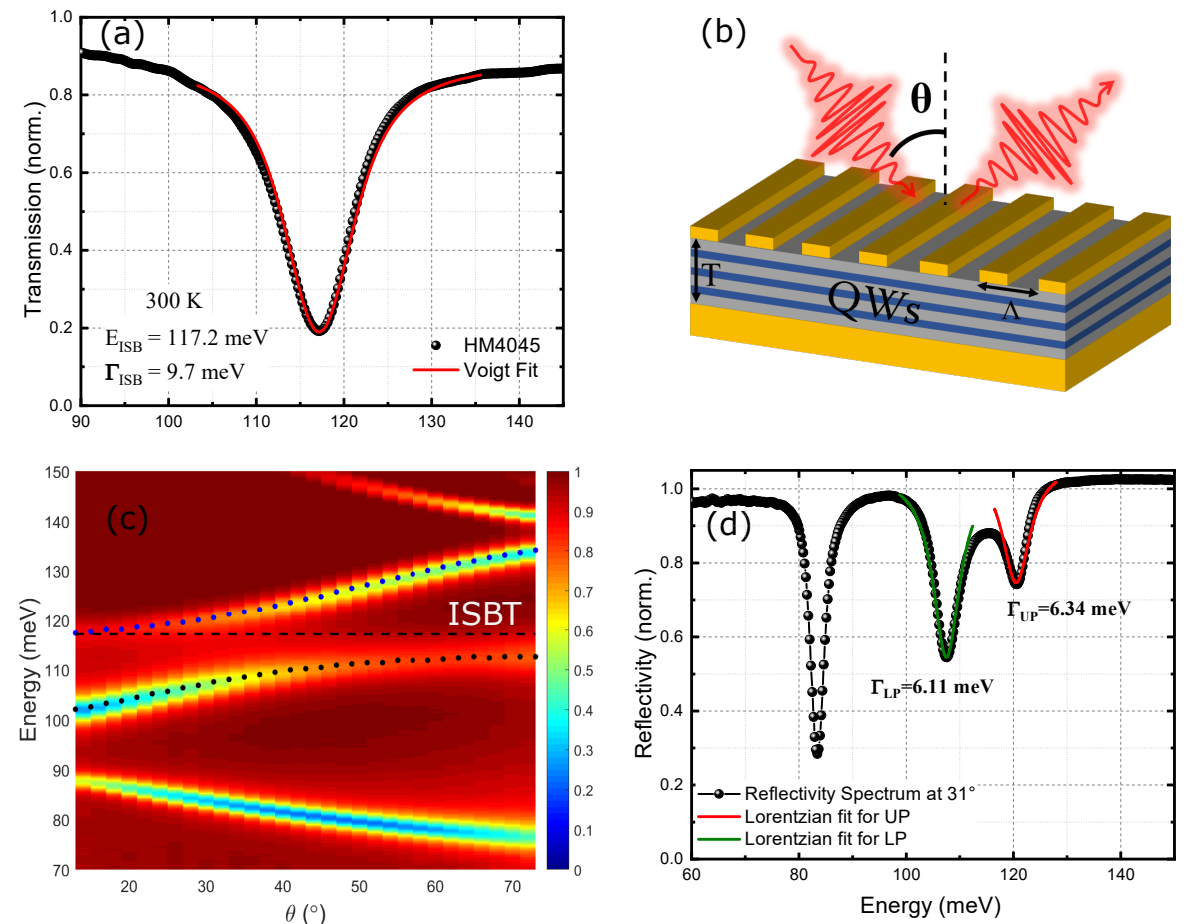

Figure 4. (a) Transmission spectrum at $300 \mathrm{~K}$ of the sample HM4045. The sample was grown with $120 \mathrm{~s}$ interruption time on the inverted interfaces. (b) The schematic of the cavity for operation in strong light matter coupling, and its angle-resolved measurement approach (c) The reconstructed angle-resolved dispersion of the HM4045 sample in the metal-metal cavity configuration. The lower and upper polaritons reflectivity minima are depicted in black and blue dots, respectively. The dashed black line shows the central frequency of the ISBT. (d) The reflectivity spectrum acquired at an angle of incidence of $31^{\circ}$ (50\%-50\% light-matter fraction). The polariton linewidths are estimated using a Lorentzian fitting procedure.

Additionally, we evaluate the influence of the growth interruption on the strong coupling regime. The polariton linewidths at zero-detuning are calculated by the equation $\left(\Gamma_{\mathrm{Cav}}+\Gamma_{+\mathrm{g} . \mathrm{i} .}\right) / 2$, where $\Gamma_{+\mathrm{g} \text {.i. }}$ 
stands for ISB absorption linewidths of samples grown by the interruption technique at inverted interfaces and by $\left(\Gamma_{\mathrm{Cav}}+\Gamma_{\mathrm{No} \text { g.i. }}\right) / 2$, for the ISB absorption linewidth of the sample without the interruption. Inserting the results from Section $2, \Gamma_{\mathrm{No} \mathrm{g.i.}}=8.2 \mathrm{meV}, \Gamma_{+ \text {g.i. }}=7.3 \mathrm{meV}$, the reduction of the polariton linewidths can go up to $9 \%$ at $78 \mathrm{~K}$. Consequently, we estimate the reduction of the theoretical threshold intensity for ISB polaritonic coherent emitters by inserting the polaritonic linewidth reductions, $\Delta \Gamma_{\mathrm{LP}} \sim \Delta \Gamma_{\mathrm{UP}} \sim 9 \%$, in Equation (1). We obtain a reduction of the threshold intensity, $\Delta I_{\text {pump }}^{\text {th }}$ of $17 \%$. These simple calculations reveal the remarkable impact of the growth interruption on the narrowing of the polariton linewidths and, in turn, on the reduction of the threshold intensity for an ISB polaritonic coherent emitter.

\section{Conclusions}

In conclusion, we have explored the two main scattering mechanisms causing the broadening of ISBT transition. We found that for relatively low doped QWs, once the donors are placed within the barrier, their influence on the broadening is minimized. We then explored the influence of growth interruption on both interfaces of multiple $\mathrm{GaAs} / \mathrm{Al}_{0.33} \mathrm{Ga}_{0.67} \mathrm{As}$ quantum wells. We observed a narrowing of ISB transitions by interruptions at GaAs-on-AlGaAs interfaces and a broadening by interruptions at AlGaAs-on-GaAs interfaces. Additionally, we have investigated the effect of the growth interruption in the strong coupling regime when embedding the heterostructure in a metal-metal grating based dispersive cavity. This approach reveals no presence of inhomogeneity within our MQW structure. Finally, we theoretically predict the reduction on both polariton linewidths $(9 \%)$ and the threshold intensity (up to $17 \%$ ) for the realization of an ISB polaritonic coherent light emitter.

Author Contributions: Conceptualization, J.-M.M.; Formal analysis, N.-L.T. and J.-M.M.; Funding acquisition, G.B., F.J. and R.C.; Investigation, N.-L.T., A.J. and A.B.; Resources, G.B.; Supervision, J.-M.M., R.C.; Writing-original draft, N.-L.T.; Writing—review \& editing, G.B., J.-M.M. and R.C.

Funding: This project has received funding from the EU-H2020 research and innovation program under grant agreement No 654360 having benefitted from the access provided by CNR-IOM in Trieste, Italy within the framework of the NFFA (Nanoscience foundries \& fine analysis) Europe Transnational Access Activity. We acknowledge financial support from the European Union FET-Open Grant MIR-BOSE (737017) and the European Research Council (IDEASERC) (“GEM") (306661). This work was partly supported by the French RENATECH network.

Conflicts of Interest: The authors declare no conflict of interest.

\section{References}

1. Faist, J.; Capasso, F.; Sivco, D.L.; Sirtori, C.; Hutchinson, A.L.; Cho, A.Y. Quantum Cascade Laser. Science 1994, 264, 553-556. [CrossRef] [PubMed]

2. Levine, B.F. Quantum-well infrared photodetectors. J. Appl. Phys. 1993, 74, R4. [CrossRef]

3. Nookala, N.; Xu, J.; Wolf, O.; March, S.; Sarma, R.; Bank, S.; Klem, J.; Brener, I.; Belkin, M. Mid-infrared second-harmonic generation in ultra-thin plasmonic metasurfaces without a full-metal backplane. Appl. Phys. A 2018, 124, 132. [CrossRef]

4. De Liberato, S.; Ciuti, C. Stimulated Scattering and Lasing of Intersubband Cavity Polaritons. Phys. Rev. Lett. 2009, 102, 136403. [CrossRef]

5. Colombelli, R.; Manceau, J.-M. Perspectives for Intersubband Polariton Lasers. Phys. Rev. X 2015, 5, 011031.

6. Campman, K.L.; Schmidt, H.; Imamoglu, A.; Gossard, A.C. Interface roughness and alloy-disorder scattering contributions to intersubband transition linewidths. Appl. Phys. Lett. 1996, 69, 2554-2556. [CrossRef]

7. Dupont, E.B.; Delacourt, D.; Papillon, D.; Schnell, J.P.; Papuchon, M. Influence of ionized impurities on the linewidth of intersubband transitions in GaAs/GaAlAs quantum wells. Appl. Phys. Lett. 1992, 60, 2121-2122. [CrossRef]

8. Faist, J.; Sirtori, C.; Capasso, F.; Pfeiffer, L.; West, K.W. Phonon limited intersubband lifetimes and linewidths in a two-dimensional electron gas. Appl. Phys. Lett. 1994, 64, 872-874. [CrossRef] 
9. Delteil, A.; Vasanelli, A.; Jouy, P.; Barate, D.; Moreno, J.C.; Teissier, R.; Baranov, A.N.; Sirtori, C. Optical phonon scattering of cavity polaritons in an electroluminescent device. Phys. Rev. B 2011, 83, 081404. [CrossRef]

10. Manceau, J.-M.; Tran, N.-L.; Biasiol, G.; Laurent, T.; Sagnes, I.; Beaudoin, G.; De Liberato, S.; Carusotto, I.; Colombelli, R. Resonant intersubband polariton-LO phonon scattering in an optically pumped polaritonic device. Appl. Phys. Lett. 2018, 112, 191106. [CrossRef]

11. Hopfield, J.J. Theory of the Contribution of Excitons to the Complex Dielectric Constant of Crystals. Phys. Rev. 1958, 112, 1555-1567. [CrossRef]

12. Manceau, J.-M.; Zanotto, S.; Sagnes, I.; Beaudoin, G.; Colombelli, R. Optical critical coupling into highly confining metal-insulator-metal resonators. Appl. Phys. Lett. 2013, 103, 91110. [CrossRef]

13. Tran, N.-L.; Malerba, M.; Talneau, A.; Biasiol, G.; Ouznali, O.; Bousseksou, A.; Manceau, J.-M.; Colombelli, R. III-V on $\mathrm{CaF}_{2}$ : A possible waveguiding platform for mid-IR photonic devices. Opt. Express 2019, 27, 1672-1682. [CrossRef] [PubMed]

14. Houdré, R.; Stanley, R.P.; Ilegems, M. Vacuum-field Rabi splitting in the presence of inhomogeneous broadening: Resolution of a homogeneous linewidth in an inhomogeneously broadened system. Phys. Rev. A 1996, 53, 2711-2715. [CrossRef] [PubMed]

15. Manceau, J.-M.; Biasiol, G.; Tran, N.L.; Carusotto, I.; Colombelli, R. Immunity of intersubband polaritons to inhomogeneous broadening. Phys. Rev. B 2017, 96, 235301. [CrossRef]

16. Manceau, J.-M.; Zanotto, S.; Ongarello, T.; Sorba, L.; Tredicucci, A.; Biasiol, G.; Colombelli, R. Mid-infrared intersubband polaritons in dispersive metal-insulator-metal resonators. Appl. Phys. Lett. 2014, 105, 081105. [CrossRef]

17. Helm, M. The basic physics of intersubband transitions. In Intersubband Transitions in Quantum Wells: Physics and Device Applications I; Liu, H.C., Capasso, F., Eds.; Elsevier Academic Press Inc.: San Diego, CA, USA, 2000; pp. 1-99.

18. Helman, A.; Tchernycheva, M.; Lusson, A.; Warde, E.; Julien, F.H.; Moumanis, K.; Fishman, G.; Monroy, E.; Daudin, B.; Dang, D.L.S.; et al. Intersubband spectroscopy of doped and undoped GaN/AIN quantum wells grown by molecular-beam epitaxy. Appl. Phys. Lett. 2003, 83, 5196. [CrossRef]

19. Ando, T.; Fowler, A.B.; Stern, F. Electronic properties of two-dimensional systems. Rev. Mod. Phys. 1982, 54, 437-672. [CrossRef]

20. Warburton, R.J.; Weilhammer, K.; Kotthaus, J.P.; Thomas, M.; Kroemer, H. Influence of Collective Effects on the Linewidth of Intersubband Resonance. Phys. Rev. Lett. 1998, 80, 2185-2188. [CrossRef]

21. Graf, S.; Sigg, H.; Köhler, K.; Bächtold, W. Direct Observation of Depolarization Shift of the Intersubband Resonance. Phys. Rev. Lett. 2000, 84, 2686-2689. [CrossRef]

22. Stormer, H. Electron Mobilities in Modulation-Doped Gaas-(Alga)As Heterostructures. Surf. Interfaces Phys. Electron. 1983, 132, 519-526.

23. Sakaki, H.; Tanaka, M.; Yoshino, J. One Atomic Layer Heterointerface Fluctuations in GaAs-AlAs Quantum Well Structures and their Suppression by Insertion of Smoothing Period in Molecular Beam Epitaxy. Jpn. J. Appl. Phys. 1985, 24, 417-420. [CrossRef]

24. Weisbuch, C.; Dingle, R.; Gossard, A.C.; Wiegmann, W. Optical characterization of interface disorder in GaAs-Ga1-xAlxAs multi-quantum well structures. Solid State Commun. 1981, 38, 709-712. [CrossRef]

25. Goldstein, L.; Horikoshi, Y.; Tarucha, S.; Okamoto, H. Effect of Well Size Fluctuation on Photoluminescence Spectrum of AlAs-GaAs Superlattices. Jpn. J. Appl. Phys. 1983, 22, 1489-1492. [CrossRef]

26. Unuma, T.; Yoshita, M.; Noda, T.; Sakaki, H.; Akiyama, H. Intersubband absorption linewidth in GaAs quantum wells due to scattering by interface roughness, phonons, alloy disorder, and impurities. J. Appl. Phys. 2003, 93, 1586. [CrossRef]

27. Tanaka, M.; Sakaki, H.; Yoshino, J. Atomic-Scale Structures of Top and Bottom Heterointerfaces in GaAs- $\mathrm{Al}_{\mathrm{x}} \mathrm{Ga}_{1-\mathrm{x}} \mathrm{As}$ (x = $0.2-1$ ) Quantum Wells Prepared by Molecular Beam Epitaxy with Growth Interruption. Jpn. J. Appl. Phys. 1986, 25 Pt 2, L155-L158. [CrossRef]

28. Sakaki, H.; Tanaka, M. Atomistic models of interface structures of $\mathrm{GaAs}_{-} \mathrm{Al}_{\mathrm{x}} \mathrm{Ga}_{1-\mathrm{x}} \mathrm{As}(\mathrm{x}=0.2-1)$ quantum wells grown by interrupted and uninterrupted MBE. J. Cryst. 1987, 81, 153-158.

29. Tu, C.; Miller, R.; Petroff, P.; Harris, T.; Kopf, R.; Sputz, S.; Lamont, M.; Wilson, B. Properties of (Al,Ga)As/GaAs heterostructures grown by molecular beam epitaxy with growth interruption. J. Cryst. 1987, 81, 159-163. [CrossRef] 
30. Petroff, P.M.; Miller, R.C.; Gossard, A.C.; Wiegmann, W. Impurity trapping, interface structure, and luminescence of GaAs quantum wells grown by molecular beam epitaxy. Appl. Phys. Lett. 1984, 44, 217. [CrossRef]

31. Singh, J.; Bajaj, K.K. Role of interface roughness and alloy disorder in photoluminescence in quantum-well structures. J. Appl. Phys. 1985, 57, 5433-5437. [CrossRef]

32. Khurgin, J.B. Inhomogeneous origin of the interface roughness broadening of intersubband transitions. Appl. Phys. Lett. 2008, 93, 91104. [CrossRef]

33. Murphy, F.J.; Bak, A.O.; Matthews, M.; Dupont, E.; Amrania, H.; Phillips, C.C. Linewidth-narrowing phenomena with intersubband cavity polaritons. Phys. Rev. B 2014, 89, 205319. [CrossRef]

2019 by the authors. Licensee MDPI, Basel, Switzerland. This article is an open access article distributed under the terms and conditions of the Creative Commons Attribution (CC BY) license (http://creativecommons.org/licenses/by/4.0/). 